\title{
A FAMÍLIA DAS BAMBUS[N]URILAS
}

\author{
Renato Salviato Cicolani\# e Grégoire Jean-François Demets* \\ Departamento de Química, Faculdade de Filosofia Ciências e Letras de Ribeirão Preto, Universidade de São Paulo, Av. Bandeirantes \\ 3900, 14040-901 Ribeirão Preto - SP, Brasil
}

Recebido em 13/12/2017; aceito em 03/05/2018; publicado na web em 19/06/2018

\begin{abstract}
THE BAMBUS[N]URIL FAMILY. This review describes the properties of the bambus[n]urils and some of their applications. These macrocycles are synthesized by the condensation of glycoluril derivatives and paraformaldehyde in acid medium. The alternate conformation of the monomers creates low electron density regions at the center of the macrocycle cavity and this is the main cause of bambus[n]urils high affinity and selectivity towards anions. Due to these properties, bambus[n]urils have been used for the detection of anions in mixtures, but also in complex supramolecular systems where they act as donors in photoinduced electron-transfer processes, and synthetic ion-channels for example. These compounds are relatively recent and deserve attention for their potential in many branches of chemistry, especially in supramolecular and inclusion chemistry.
\end{abstract}

Keywords: cavitands; bambus[n]urils; anion binding; inclusion chemistry.

\section{INTRODUÇÃO}

Macrociclos orgânicos que delimitam cavidades representam uma oportunidade ímpar para o estudo da química em meio confinado, que é um ramo muito importante da química supramolecular. A associação desses macrociclos com outras moléculas é consequência de interações não covalentes entre moléculas hóspedes-hospedeiras ${ }^{1}$ e tem sido muito estudada por conta de diversas aplicações em catálise, estocagem e transporte de fármacos e sensores, por exemplo. ${ }^{2}$ Cavitandos como as cucurbit[n]urilas $(\mathbf{C B}[\mathbf{n}]),{ }^{3-6}$ pirogalol[n]arenos, ${ }^{7}$ calix[n] arenos, ${ }^{8}$ ou as ciclodextrinas, ${ }^{9}$ por exemplo, possuem cavidades capazes de hospedar outras entidades químicas denominadas hóspedes. A restrição vibracional imposta pelo macrociclo (hospedeiro) somada a um campo elétrico particular existente em sua cavidade pode levar a molécula hóspede a estados energéticos e configurações (vibracional, rotacional e/ou eletrônico) diferenciados, até então insustentáveis em solução ou fase gasosa, a ponto de alterar consideravelmente suas propriedades físico-químicas assim como sua reatividade. ${ }^{10}$

As bambus[n]urilas, abreviadas por BU[n] $(\mathrm{n}=4,6)$, são uma classe de macrociclos análogas às cucurbiturilas que foram sintetizadas pela primeira vez por Sindelar e colaboradores ${ }^{11}$ a partir da reação de condensação da 2,4-dimetilglicolurila com paraformaldeído em meio ácido. Os autores verificaram que o produto obtido, a metil-bambus[6]urila (MeBU[6]), apresentava conformação similar à de um gomo de bambu, planta da família bambusoideae, da qual emprestou seu nome. É importante ressaltar que as glicolurilas utilizadas na síntese das BU[n] são formadas a partir de uma ureia não substituída e outra contendo substituinte em seus nitrogênios. Isso faz com que os monômeros das $\mathbf{B U}[\mathbf{n}]$ sejam unidos por uma ponte metilênica apenas e se arranjem de forma alternada nos macrociclos (Figura 1). Esta conformação peculiar gera uma cavidade com baixa densidade eletrônica, que justifica a grande afinidade e seletividade das BU[n] por ânions e hóspedes com alta densidade eletrônica. ${ }^{12}$

Por conta desta característica, as BU[n] vêm ganhando espaço na literatura especializada, e têm se mostrado importantes blocos de montagem em sistemas supramoleculares diversos, para fins de desenvolvimento de sensores, ${ }^{13}$ translocação de ânions através de

*e-mail: greg@usp.br

\#alternative e-mail: renatosc@usp.br

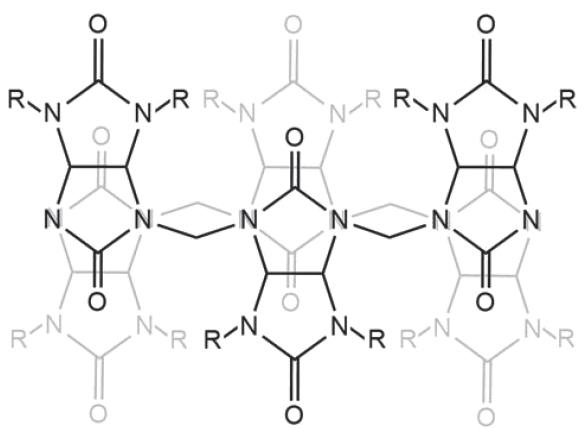

Figura 1. Fórmula estrutural das bambus[n]urilas, salientando sua conformação alternada

membranas lipídicas, ${ }^{14}$ como molde em estruturas auto-organizadas ${ }^{15}$ e na detecção de ânions em solução aquosa. ${ }^{16} \mathrm{~A}$ presente revisão tem como objetivo apresentar a família das bambus[n]urilas, sua síntese, suas propriedades físico-químicas mais importantes e algumas de suas aplicações mais relevantes e atuais.

\section{SÍNTESE E PROPRIEDADES}

A primeira síntese de $\mathbf{B U}[\mathbf{n}]$ descrita na literatura, ${ }^{11}$ a da MeBU[6], envolve a preparação de dois precursores, a trans-4,5-di-hidroxi-imidazolidin-2-ona e a 2,4-dimetilglicolurila (Figura 2). Partindo de ureia e glioxal em meio alcalino ${ }^{17}$ se obtém a trans-4,5-di-hidroxi-imidazolidin-2-ona, que reage posteriormente com a 1,3-dimetilureia em meio ácido para produzir a 2,4-dimetilglicolurila. A reação da 2,4-dimetilglicolurila com paraformaldeído em ácido clorídrico produz uma mistura que contém polímeros lineares e uma mistura dos macrociclos MeBU[4] e MeBU[6]. Após as etapas de purificação, o produto majoritário isolado é na realidade o caviplexo $\mathrm{H}^{+}\left(\mathrm{Cl}\right.$ @ MeBU[6]) ou simplesmente $\mathrm{Cl}^{-} @$ MeBU[6]. É possível ainda obter o macrociclo vazio MeBU[6 $]^{12}$ tratando o caviplexo $\mathrm{Cl}^{-} @$ MeBU[6] com ácido iodídrico para substituir o íon cloreto por iodeto e posteriormente eliminá-lo na forma de iodo molecular por oxidação com peróxido de hidrogênio.

Algo interessante é que as cucurbit[n]urilas $(\mathbf{C B}[\mathbf{n}])$, que são estruturalmente análogas às $\mathbf{B U}[\mathbf{n}]$, também são obtidas a partir da reação de condensação de uma glicolurila com formaldeído em meio 


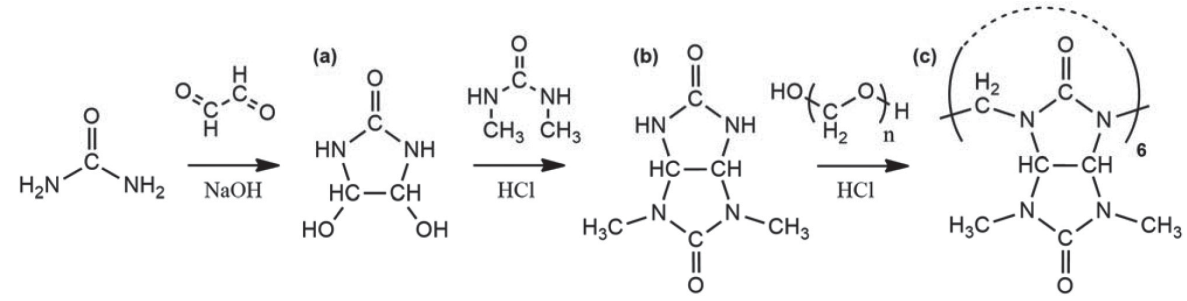

Figura 2. Esquema de síntese para obtenção da (a) 4,5-di-hidroxi-imidazolidin-2-ona, (b) 2,4-dimetilglicolurila e (c) metil-bambus[6]urila

ácido. ${ }^{4}$ No entanto, o arranjo diferente das unidades de glicolurila na estrutura das $\mathbf{C B}[\mathbf{n}]$ faz com que formem complexos com cátions e moléculas neutras. As hemi-cucurbit[n]urilas (hmCB[n]), ${ }^{18,19}$ outro tipo de macrociclo hospedeiro, são formadas a partir de grupamentos imidazolidin-2-ona conectados por pontes metilênicas. Basicamente, a estrutura das $\mathbf{h m C B}[\mathbf{n}]$ é similar a uma $\mathbf{C B}[\mathbf{n}]$ cortada simetricamente pelo plano equatorial. Como as unidades imidazolin-2-ona das hmCB[n] são ligadas somente por uma ponte metilênica, a estrutura desses macrociclos é pouco rígida e permite a alternância dos monômeros, da mesma forma que ocorre com as $\mathbf{B U}[\mathbf{n}]$. Isso ocasiona na formação de uma região com menor densidade eletrônica no centro da molécula, fazendo com que as $\mathbf{h m C B}[\mathbf{n}]$ e as BU[n] apresentem maior afinidade por ânions. Comparando a estrutura da MeBU[6], da hmCB[6] e da CB[6] é possível verificar que o arranjo espacial dos monômeros e sobretudo sua liberdade para adotarem ou não a configuração alternada são fatores preponderantes nas propriedades das cavidades que delimitam, como solvofobicidade e seletividade (Figura 3). ${ }^{20}$

Ainda que as hmCB[n] complexem ânions, a afinidade e seletividade com que o fazem são consideravelmente menores que as das BU[n], o que se explica pela diferença de densidade eletrônica superficial das bambusurilas. A inclusão de haletos na cavidade da MeBU[6] ocorre por conta de interações entre os átomos de hidrogênio da cintura do macrociclo com o haleto, gerando ligações de hidrogênio do tipo C-H $\cdots X$. A MeBU[6] apresenta um diâmetro de $6,4 \AA$ e $12,7 \AA$ de comprimento, ${ }^{11}$ o que torna a cavidade relativamente grande para inclusão e estabilização de outras moléculas (Figura 4). Sindelar e colaboradores ${ }^{21}$ determinaram a constante de afinidade da MeBU[6] e haletos por meio de titulações microcalorimétricas e confirmaram a tendência observada anteriormente, ${ }^{11,12}$ sendo a constante de afinidade da MeBU[6] pelos íons iodeto e brometo cerca de 1000 e 100 vezes maior, respectivamente, que por aquela observada para íon cloreto.

$\mathrm{O}$ espectro de RMN de ${ }^{1} \mathrm{H}$ da MeBU[6] é relativamente simples, ${ }^{12}$ sendo observados apenas três sinais, em $\delta=5,41 \mathrm{ppm}$ (s, 12H), 4,20 ppm (s, 12H) e 3,03 ppm (s, 36H) que são atribuídos aos hidrogênios da cintura, da ponte metilênica e do substituinte metila, respectivamente. A presença de ânions no interior da cavidade da MeBU[6] influencia no deslocamento químico destes átomos de hidrogênio, ${ }^{11}$ como por exemplo no caso do caviplexo $\mathrm{Cl}^{-} @$ MeBU[6] que apresenta deslocamentos químicos em 5,35, 5,15 e 3,11 ppm.

Os dados sobre as propriedades físico-químicas fundamentais da MeBU[6] ainda são relativamente escassos na literatura, mas consideráveis avanços tem sido realizados por Cicolani, ${ }^{22}$ tais como determinação do comportamente térmico, solubilidade em diversos solventes e formação de complexos com cátions. A estabilidade térmica da MeBU[6] não é discutida na literatura, somente é apresentado que o caviplexo $\mathrm{Cl}^{-} @ \mathbf{M e B U}$ [6] sofre degradação em temperaturas acima de $300{ }^{\circ} \mathrm{C} .{ }^{11} \mathrm{Com}$ relação aos dados de solubilidade presentes na literatura, a MeBU[6] é muito pouco solúvel na maioria dos solventes usuais, sendo que em água, a solubilidade do caviplexo
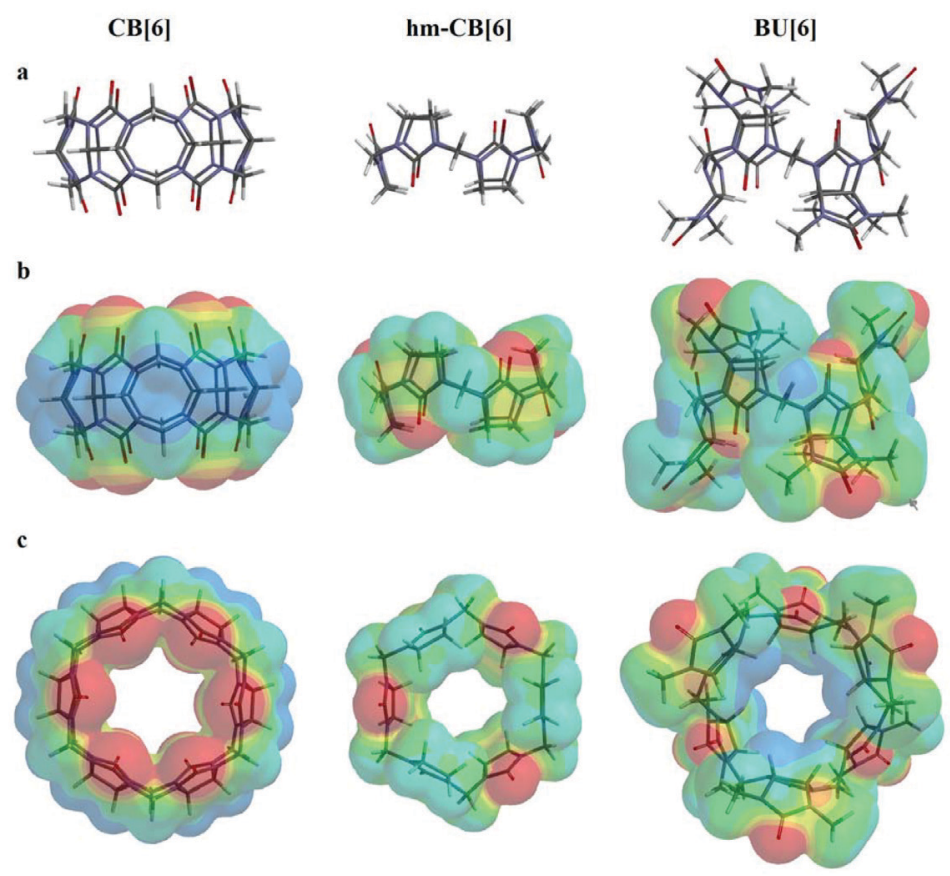

Figura 3. (a) Visão lateral dos macrociclos CB[6] (esquerda), hmCB[6] (centro) e BU[6] (direita). Mapa de densidade superficial de elétrons dos mesmos macrociclos, visto (b) de lado e (c) por cima. As superfícies foram calculados por DFT a nível de teoria B3LYP/6-31G*. As regiões em vermelho e azul representam, respectivamente, regiões de maior e menor densidade eletrônica 
(a)

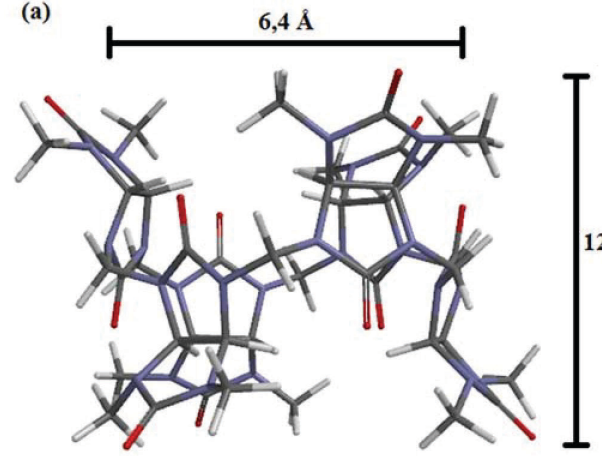

(b)

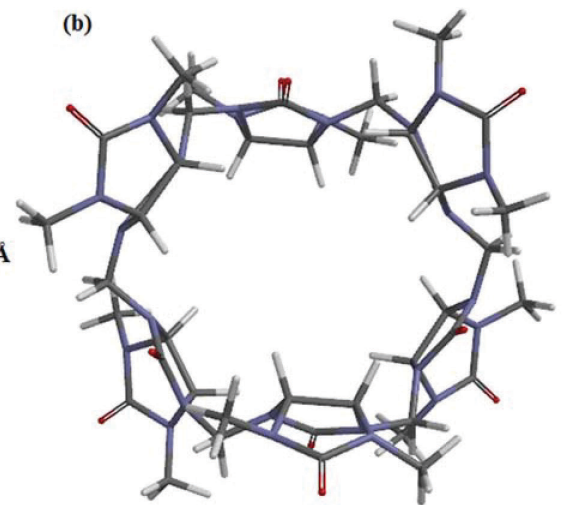

Figura 4. (a) Visão lateral da MeBU[6] indicando seu diâmetro e comprimento. (b) Visão superior da MeBU[6]

$\mathrm{Cl}^{-} @$ MeBU[6] é de apenas $0,020 \mathrm{~g} \mathrm{dm}^{-3}$, enquanto que a MeBU[6] é totalmente insolúvel. ${ }^{11,12}$

Cálculos computacionais foram realizados para prever e elucidar algumas das propriedades fundamentais da MeBU[6]. O espectro de infravermelho simulado para a MeBU[6], ${ }^{23}$ por exemplo, mostra que este macrociclo apresenta diversos modos vibracionais, sendo que se destacam as bandas em 1757 e $1714 \mathrm{~cm}^{-1}$, atribuídas ao estiramento das ligações $\mathrm{C}=\mathrm{O}$ do grupamento 1,3-dimetilureia e ureia, respectivamente, em $1168 \mathrm{~cm}^{-1}$ atribuída ao estiramento da ligação C-C e em 775 $\mathrm{cm}^{-1}$ atribuída ao estiramento da ligação C-N-C da ponte metilênica.

Diversos cálculos foram realizados para prever a formação de caviplexos envolvendo a MeBU[6] e ânions, como $\mathrm{ClO}_{4}{ }^{-}$e $\mathrm{BF}_{4}{ }^{-24}$ $\mathrm{CN}^{-},{ }^{25} \mathrm{OCN}^{-}$e SCN-$,{ }^{26} \mathrm{NO}_{3},{ }^{27} \mathrm{HS}^{-},{ }^{28}$ metanossulfonato e trifluorometanossulfonato. ${ }^{29}$ Existem também cálculos envolvendo a complexação da MeBU[6] com os cátions $\mathrm{H}_{3} \mathrm{O}^{+},{ }^{30} \mathrm{Cs}^{+31} \mathrm{e} \mathrm{Na}^{+}{ }^{32}$ nos átomos de oxigênio dos opérculos. Vale ressaltar que em todas as simulações com cátions foram utilizados pares iônicos, uma vez que as interações com cátions sozinhos não são termodinamicamente favoráveis.

Desde a descoberta da MeBU[6], diversos derivados foram reportados contendo resíduos alifáticos e substituintes aromáticos. ${ }^{33-37} \mathrm{De}$ maneira geral, as glicolurilas dissubstituídas reagem com formaldeído em meio ácido, em condições específicas, produzindo as respectivas BU[n] funcionalizadas (Figura 5). As benzil-bambus[4]urila (BnBU[4]), benzil-bambus[6]urila (BnBU[6]) e propil-bambus[6] urila (PrBU[6] $)^{34}$ foram obtidas a partir das 2,4-dibenzilglicolurila ou 2,4-dipropilglicolurila, respectivamente, em solventes apolares, e não em meio aquoso como na síntese da MeBU[6]. A BnBU[6] obtida era capaz de formar complexos com elevada constante de formação com diversos ânions em clorofórmio, ${ }^{35}$ diferente da MeBU[6], que é pouco solúvel nesse mesmo solvente. Esse resultado mostrou a influência do substituinte ligado às $\mathbf{B U}[\mathbf{n}]$ nas propriedades da mesma.

Heck e colaboradores ${ }^{36}$ obtiveram os derivados alil-bambus[4] urila (AlilBU[4]) e alil-bambus[6]urila (AlilBU[6]) a partir de síntese assistida por micro-ondas, utilizando a di-alil-glicolurila como precursor. Alterações nas condições de síntese levavam a diferentes distribuições de homólogos. Além disso, os autores obtiveram a PrBU[4] a partir da hidrogenação catalítica da AlilBU[4] utilizando $\mathrm{H}_{2}$ na presença de catalisador $10 \% \mathrm{Pd} / \mathrm{C}$ em etanol.

Sindelar e colaboradores sintetizaram a $p$-carboxi-benzil-bambus[6]urila (BzCOOH)BU[6], ${ }^{37}$ de elevada solubilidade em meio aquoso neutro e básico, e as 3-carboxipropil-bambus[6]urila (PrCOOH)BU[6] e 5-carboxipentil-bambus[6]urila (pentilCOOH) BU[6]. ${ }^{38} \mathrm{~A}$ afinidade por ânions destes últimos é modulada tanto pelo tamanho do substituinte R ligado à BU[6] quanto pelo $\mathrm{pH}$ da solução. A obtenção de derivados das BU[n] solúveis em água e com afinidade por ânions representa um passo importante para o estudo destes cavitandos, uma vez que permite contornar limitações óbvias no que tange às aplicações, como em bioquímica e medicina, ${ }^{2,33}$ por exemplo, e foram assunto de investigações recentes.

Além de BU[n] modificadas com diversos grupos laterais R, foram sintetizados outros derivados por substituição do átomo de oxigênio do grupamento 1,3-dimetilureia do macrociclo (Figura 6).

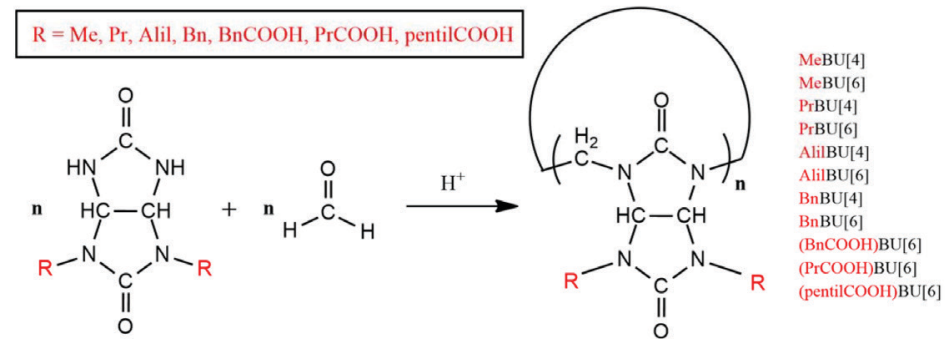

Figura 5. Esquema geral de síntese das bambus[n]urilas $(n=4,6)$ a partir da condensação de glicolurilas dissubstituídas com formaldeído catalisada por ácido
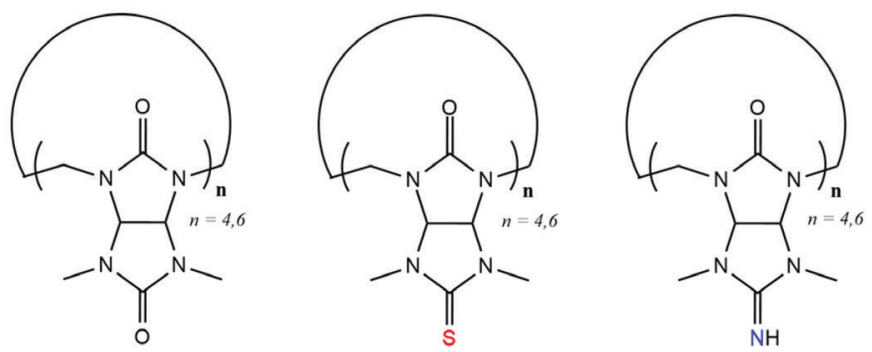

Figura 6. Estrutura das MeBU[6] (esquerda), MeBUS[6] (centro) e MeBUN[6] (direita) 
As metil-semitio-bambus[4]urila (MeBUS[4]) e metil-semitio-bambus[6]urila (MeBUS[6]), ${ }^{39}$ por exemplo, são obtidas a partir da reação da 2,4-dimetil-semitio-glicolurila com paraformaldeído em meio ácido. Um ponto interessante é que esses derivados das BU[n] são capazes de incluir ânions em seu interior e complexar

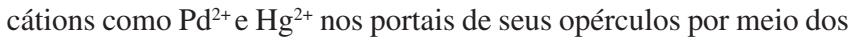
átomos de enxofre.

Outros exemplos de substituição do hetero-átomo do grupamento 1,3-dimetilureia da $\mathbf{B U}[\mathbf{n}]$ reportado na literatura são as metil-semiaza-bambus[4]urila (MeBUN[4]) e metil-semiaza-bambus[6]urila (MeBUN[6]), ${ }^{40,41}$ macrociclos obtidos a partir da reação das MeBUS[4] e MeBUS[6] com aminas em refluxo de tetrahidrofurano. Estas semiaza-bambusurilas, são capazes de complexar múltiplos ânions em suas cavidades como, por exemplo, dois íons trifluorometanossulfonato e um íon iodeto simultaneamente. Ao mesmo tempo que as MeBUN[4] e MeBUN[6] são capazes de formar caviplexos relativamente estáveis com ânions, estes são lábeis o suficiente para desligarem-se deles em determinadas condições e permitir seu trânsito pelas cavidades, evidenciando assim seu potencial como ionóforos sintéticos.

Titulações calorimétricas isotérmicas (ITC) e de RMN de ${ }^{1} \mathrm{H}$ foram efetuadas para obtenção das constantes de formação dos caviplexos $\left(K_{\text {form }}\right)$ de BU[n] com diversos ânions (Tabela 1). A partir dos resultados obtidos, é possível verificar que o tamanho e simetria do ânion caviplexado influenciam na afinidade, bem como o substituinte ou hetero-átomo do macrociclo.

\section{ALGUMAS APLICAÇÕES}

\section{Detecção de ânions em mistura por $\mathbf{R M N}$ de $^{1} \mathrm{H}$}

Sensores químicos seletivos a analitos específicos são importantes para detecção e quantificação de cátions, ${ }^{42}$ ânions ${ }^{43}$ ou moléculas neutras. ${ }^{44}$ Existe uma grande demanda por sistemas químicos capazes de identificar ânions em solução como por exemplo, em análises de água e análises ambientais em geral. ${ }^{45} \mathrm{~A}$ maioria dos sensores para ânions descritos na literatura opera em meio orgânico, sendo ainda escassos aqueles que funcionem em água. ${ }^{46,47}$

Dada a capacidade das BU[n] de incluir ânions seletivamente, Sindelar e seu grupo ${ }^{48}$ adicionaram os derivados BnBU[6] (DMSO- $\mathrm{d}_{6}$ $+5 \%$ de $\left.\mathrm{D}_{2} \mathrm{O}\right)$ e (BnCOOH)BU[6] $\left(\mathrm{K}_{2} \mathrm{DPO}_{4} 20 \mathrm{mmol} \mathrm{dm}^{-3} \mathrm{em} \mathrm{D}_{2} \mathrm{O}\right)$, respectivamente, a soluções que continham doze ânions distintos e realizaram análises de $\mathrm{RMN}$ de ${ }^{1} \mathrm{H}$ na tentativa de utilizar esses macrociclos como sensores de ânions.

Os espectros de RMN de ${ }^{1} \mathrm{H}$ indicaram a formação de caviplexos com estequiometria de 1:1 e os deslocamentos químicos observados permitiram a identificação dos respectivos ânions $\left(\mathrm{BF}_{4}^{-}, \mathrm{PF}_{6}^{-}, \mathrm{ClO}_{4}^{-}\right.$, $\mathrm{NO}_{3}^{-}, \mathrm{ReO}_{4}^{-}, \mathrm{CN}^{-}, \mathrm{HSO}_{4}^{-} \mathrm{Cl}^{-}, \mathrm{Br}^{-}, \mathrm{I}^{-}, \mathrm{SCN}^{-}, \mathrm{IO}_{4}^{-}$), confrontando os resultados com experimentos controle com os caviplexos de ânions isolados.

A BnBU[6] apresenta elevada seletividade em complexar com os ânions, sendo que a maioria destes pôde ser identificada pelo simples deslocamento químico dos hidrogênios da ponte metilênica (Tabela 2). Por outro lado, a (BnCOOH)BU[6], que é mais solúvel em água, apresenta menor afinidade pelos ânions $\mathrm{Cl}^{-}, \mathrm{CN}^{-}, \mathrm{IO}_{4}^{-}, \mathrm{ReO}_{4}$ e possibilita somente a diferenciação de quatro dos doze ânions em mistura somente $\left(\mathrm{PF}_{6}^{-}, \mathrm{BF}_{4}^{-}, \mathrm{ClO}_{4}^{-}, \mathrm{I}^{-}\right)$.

\section{Transferência eletrônica foto-induzida entre BU[6] e aceptor de elétrons}

Reações de transferência eletrônica foto-induzida (TEF) são a peça central de sistemas de conversão de energia luminosa em energia elétrica em sistemas químicos, como células solares, por exemplo. ${ }^{49}$
Tabela 1. Constantes de formação de caviplexos de algumas BU[n] com ânions ${ }^{21,35,37,39,40}$

\begin{tabular}{|c|c|}
\hline Ânion & $K_{\text {form }}\left(\mathrm{dm}^{3} \mathrm{~mol}^{-1}\right)$ \\
\hline \multicolumn{2}{|c|}{$\operatorname{MeBU}[6]^{a}$} \\
\hline $\mathrm{Cl}^{-}$ & $3,8 \times 10^{5}$ \\
\hline $\mathrm{Br}$ & $4,0 \times 10^{7}$ \\
\hline $\mathrm{I}^{-}$ & $4,0 \times 10^{8}$ \\
\hline \multicolumn{2}{|c|}{$\mathrm{BnBU}[6]^{\mathrm{b}}$} \\
\hline $\mathrm{CF}_{3} \mathrm{SO}_{3}^{-}$ & $2,1 \times 10^{5}$ \\
\hline $\mathrm{SbF}_{6}^{-}$ & $2,6 \times 10^{5}$ \\
\hline $\mathrm{CH}_{3} \mathrm{CO}_{2}^{-}$ & $5,6 \times 10^{5}$ \\
\hline $\mathrm{CH}_{3} \mathrm{SO}_{3}^{-}$ & $7,3 \times 10^{5}$ \\
\hline $\mathrm{F}^{-}$ & $1,9 \times 10^{6}$ \\
\hline $\mathrm{CN}^{-}$ & $9,8 \times 10^{6}$ \\
\hline $\mathrm{Cl}^{-}$ & $1,3 \times 10^{7}$ \\
\hline $\mathrm{IO}_{4}^{-}$ & $1,6 \times 10^{7}$ \\
\hline $\mathrm{ReO}_{4}^{-}$ & $1,1 \times 10^{8}$ \\
\hline $\mathrm{HSO}_{4}^{-}$ & $3,5 \times 10^{8}$ \\
\hline $\mathrm{PF}_{6}^{-}$ & $4,5 \times 10^{8}$ \\
\hline $\mathrm{Br}$ & $6,7 \times 10^{8}$ \\
\hline $\mathrm{NO}_{3}^{-}$ & $8,7 \times 10^{8}$ \\
\hline $\mathrm{BF}_{4}^{-}$ & $1,8 \times 10^{9}$ \\
\hline $\mathrm{ClO}_{4}^{-}$ & $1,0 \times 10^{10}$ \\
\hline $\mathrm{SCN}^{-}$ & $2,1 \times 10^{10}$ \\
\hline $\mathrm{I}^{-}$ & $2,6 \times 10^{10}$ \\
\hline \multicolumn{2}{|c|}{$(\mathrm{BnCOOH}) \mathrm{BU}[6]^{\mathrm{c}}$} \\
\hline $\mathrm{F}^{-}$ & $1,1 \times 10^{2}$ \\
\hline $\mathrm{Cl}^{-}$ & $9,1 \times 10^{2}$ \\
\hline $\mathrm{CN}^{-}$ & $1,1 \times 10^{3}$ \\
\hline $\mathrm{IO}_{4}^{-}$ & $6,5 \times 10^{3}$ \\
\hline $\mathrm{ReO}_{4}^{-}$ & $3,0 \times 10^{4}$ \\
\hline $\mathrm{Br}^{-}$ & $1,4 \times 10^{5}$ \\
\hline $\mathrm{NO}_{3}^{-}$ & $4,8 \times 10^{5}$ \\
\hline $\mathrm{PF}_{6}^{-}$ & $2,2 \times 10^{6}$ \\
\hline $\mathrm{BF}_{4}^{-}$ & $4,3 \times 10^{6}$ \\
\hline $\mathrm{I}^{-}$ & $1,0 \times 10^{7}$ \\
\hline $\mathrm{ClO}_{4}^{-}$ & $5,5 \times 10^{7}$ \\
\hline \multicolumn{2}{|c|}{$\operatorname{MeBUS}[6]^{\mathrm{d}}$} \\
\hline $\mathrm{CH}_{3} \mathrm{CO}_{2}^{-}$ & $2,7 \times 10^{4}$ \\
\hline $\mathrm{Cl}^{-}$ & $7,2 \times 10^{4}$ \\
\hline $\mathrm{BF}_{4}^{-}$ & $1,5 \times 10^{5}$ \\
\hline $\mathrm{I}^{-}$ & $2,6 \times 10^{5}$ \\
\hline $\mathrm{NCN}^{-}$ & $7,6 \times 10^{5}$ \\
\hline $\mathrm{Br}$ & $9,5 \times 10^{5}$ \\
\hline \multicolumn{2}{|c|}{$\operatorname{MeBUN}[6]^{\mathrm{e}}$} \\
\hline $\mathrm{TfO}^{-}$ & $5,2 \times 10^{4}$ \\
\hline $\mathrm{Br}$ & $2,9 \times 10^{5}$ \\
\hline $\mathrm{I}^{-}$ & $5,3 \times 10^{6}$ \\
\hline
\end{tabular}

a (ITC, $\left.\mathrm{CH}_{3} \mathrm{OH} / \mathrm{CHCl}_{3} 30: 70 \mathrm{v} / \mathrm{v}, 298 \mathrm{~K}\right), \mathrm{b}\left(\mathrm{ITC}, \mathrm{CHCl}_{3}, 298 \mathrm{~K}\right), \mathrm{c}(\mathrm{RMN} \mathrm{de}$ ${ }^{1} \mathrm{H}, 20 \mathrm{mmol} \mathrm{dm}^{-3} \mathrm{~K}_{2} \mathrm{DPO}_{4}$ em $\mathrm{D}_{2} \mathrm{O} \mathrm{pD} 7,1,303 \mathrm{~K}$ ), d (RMN de ${ }^{1} \mathrm{H}$, DMSO $\left.\mathrm{d}_{6}\right)$, e (ITC, $\left.\mathrm{CH}_{3} \mathrm{OH} / \mathrm{CHCl}_{3} 30: 70 \mathrm{v} / \mathrm{v}, 298 \mathrm{~K}\right)$. 
Tabela 2. Deslocamento químico ( $\delta$ ) do hidrogênio metilênico da BnBU[6] vazia e complexada a ânions diversos $\left(500 \mathrm{MHz}, \mathrm{DMSO} \mathrm{d}_{6}+5 \% \mathrm{D}_{2} \mathrm{O}\right.$, $\left.30{ }^{\circ} \mathrm{C}, \mathrm{TMS}\right)^{48}$

\begin{tabular}{cc}
\hline $\mathrm{Ânion}^{-}$ & $\delta(\mathrm{ppm})$ \\
\hline $\mathrm{BF}_{4}^{-}$ & 5,184 \\
$\mathrm{PF}_{6}^{-}$ & 5,324 \\
$\mathrm{ClO}_{4}^{-}$ & 5,353 \\
$\mathrm{NO}_{3}^{-}$ & 5,380 \\
$\mathrm{ReO}_{4}^{-}$ & 5,437 \\
$\mathrm{CN}^{-}$ & 5,493 \\
$\mathrm{HSO}_{4}^{-}$ & 5,514 \\
$\mathrm{Cl}^{-}$ & 5,559 \\
$\mathrm{Br}^{-}$ & 5,604 \\
$\mathrm{SCN}^{-}$ & 5,609 \\
$\mathrm{I}^{-}$ & 5,635 \\
$\mathrm{IO}_{4}^{-}$ & 5,668 \\
\hline
\end{tabular}

Os viologênios são dicátions orgânicos passíveis de redução por TEF e podem ser utilizados para conversão de energia solar em eletricidade.$^{50}$ No entanto, a baixa estabilidade dos radicais-cátions radicalares favorece muito decaimentos não-radiativos, dificultando sua aplicação em células solares. Por conta disso, matrizes como zeólitas $^{51}$ e redes metalorgânicas ${ }^{52}$ são utilizadas na tentativa de estabilizar esses cátions radicalares.

Recentemente, Sindelar e seu grupo ${ }^{53}$ reportaram a obtenção de um material cristalino fotoativo constituído de MeBU[6] e hexafluorofosfato de metil-viologênio, $\mathbf{M V}\left(\mathrm{PF}_{6}\right)_{2}$, em que o macrociclo age como doador de elétron na fotorredução do $\mathbf{M V}^{2+}$. A MeBU[6] complexa um íon $\mathrm{PF}_{6}-$ em cada portal, de forma que um átomo de flúor axial de cada ânion fique incluído na cavidade do macrociclo por conta de pseudo-ligações de hidrogênio do tipo C-H...F. Na forma cristalina, as camadas contendo o complexo $2 \mathrm{PF}_{6}$-@ MeBU[6] alternam-se com camadas formadas pelos íons $\mathbf{M V}^{2+}$. A estrutura resultante (Figura 7) é mantida coesa por interações coulômbicas

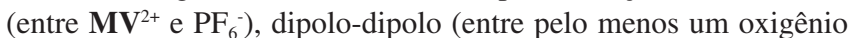
da MeBU[6] e um anel aromático do $\left.\mathbf{M V}^{2+}\right)$ e íon-dipolo $\left(\mathbf{M V}^{2+}\right.$ ou $\mathrm{PF}_{6}-\mathrm{e}$ os solventes).

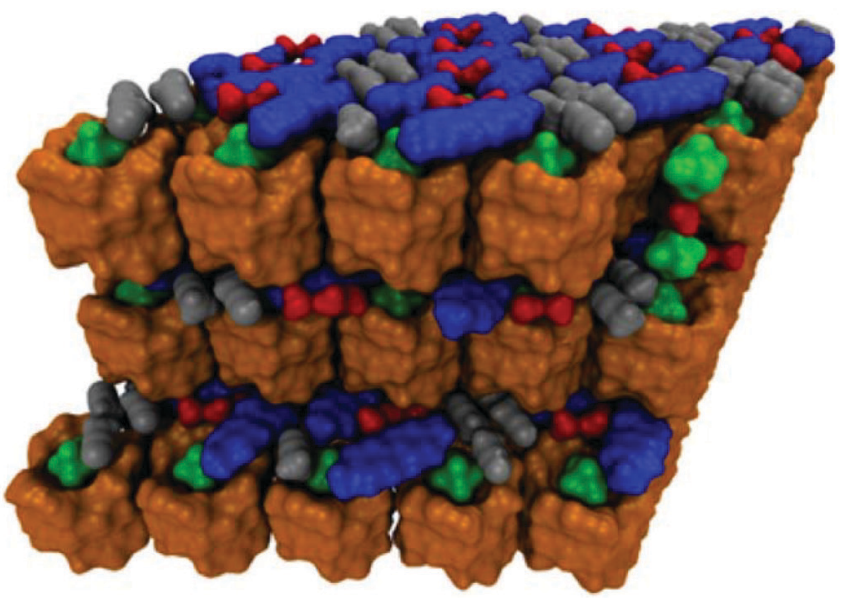

Figura 7. Empacotamento da estrutura cristalina do sistema MeBU[6]/MV ${ }^{2+}$ $P_{6}^{-}$(bambusurila: marrom; $M^{2+}$ : azul; $P F_{6}:$ verde; acetonitrila: cinza; água: vermelho). Reproduzido parcialmente com permissão da American Chemical Society (2017, v. 139, p. 2598)
A irradiação desse sólido misto de MeBU[6]/MV ${ }^{2+} / \mathrm{PF}_{6}^{-}$(Figura 8) com luz ultravioleta (266 e $375 \mathrm{~nm}$ ) leva à mudança de coloração de amarelo para azul, persistente por aproximadamente cinco horas. Esta variação de cor é indicativo típico da formação do cátion radical $\mathbf{M V}^{+\bullet}$ que só poderia ser explicado pela oxidação da MeBU[6] pelo $\mathbf{M V}^{2+}$. Os autores sugerem que, inicialmente, o viologênio do complexo $\mathbf{M V}^{2+} \cdot 2 \mathrm{PF}_{6}$-@MeBU[6] é excitado a um estado singleto com energia suficiente para oxidar a MeBU[6] e reduzir o $\mathbf{M V}^{2+}$. O par íon-radical $\mathbf{M V}^{+\bullet} \cdot 2 \mathrm{PF}_{6}$-@ MeBU[6 $]^{\bullet}$ formado no estado singleto sofre decaimento para um estado triplete de menor energia, que é responsável pela coloração azul. Finalmente, ocorre uma transferência eletrônica proibida por spin pouco eficiente, o que resulta na formação do complexo inicial $\mathbf{M V}^{2+} \cdot 2 \mathrm{PF}_{6}^{-} @ \mathbf{M e B U}[6]$ e explica o elevado tempo de meia-vida observado. Este estudo foi um marco, pois foi o primeiro do gênero envolvendo MeBU[6]. ${ }^{47}$

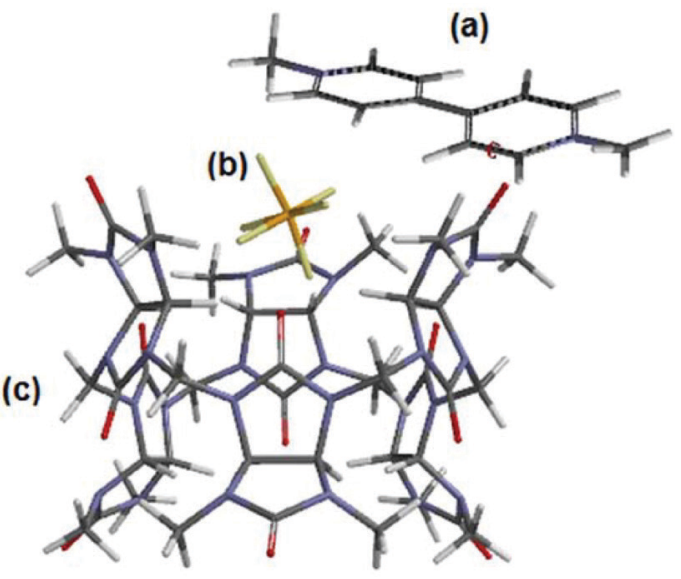

Figura 8. Complexo de metil-viologênio (a), hexafluorofosfato (b) e metil-bambus[6]urila (c), passível de transferência de elétrons foto-induzida do macrociclo para o metil-viologênio

\section{Transporte transmembrana de ânions}

A troca de ânions entre uma célula e sua vizinhança é importante para a regulação de fenômenos biológicos essenciais à vida, ${ }^{54}$ como a manutenção da concentração de íons fluoreto para crescimento de ossos e dentes, ${ }^{55}$ de íons cloreto nos rins ${ }^{56}$ e de íons iodeto nas glândulas tireóide, entre outros ${ }^{57}$ Os transportadores naturais mais comuns são proteínas de membrana ${ }^{58}$ e complexos macromoleculares. ${ }^{59}$ Muito tem sido feito para se desenvolver transportadores de ânions sintéticos, que possam agir a partir de interações eletrostáticas, ligações de hidrogênio e halogênio e/ou interações $\pi \cdot{ }^{60}$ É importante ressaltar, no entanto, que nem todos os transportadores de íons sintéticos são eficazes em sistemas biológicos, uma vez que parâmetros como o coeficiente de partição e a mobilidade no interior da membrana do transportador são propriedades essenciais a serem consideradas. ${ }^{61}$

Tendo isso em vista, Reany e colaboradores ${ }^{62}$ estudaram se as MeBU[6], MeBUS[6] e MeBUN[6] apresentavam potencial como transportadores de ânions através de bicamadas lipídicas, justamente porque os três macrociclos apresentam afinidade por ânions similares. De acordo com a literatura, ${ }^{39,40}$ a afinidade com que os macrociclos complexam o íon cloreto é MeBUS[6] > MeBU[6] > MeBUN[6]. Os autores estudaram o transporte de íons cloreto através de vesículas de 1-palmitoil-2-oleoil-sn-glicero-3-fosfocolina (POPC), monitorando a fluorescência da lucigenina, um corante sensível a ânions (Figura 9). Os resultados comprovaram que a eficiência do transporte aumentava de acordo com o aumento da afinidade dos macrociclos pelo ânion. A questão de se a MeBUS[6] poderia operar como um canal aniônico ou carreador de ânions foi resolvida a partir da adição de colesterol na 
vesícula de POPC. Os resultados indicaram que houve uma diminuição do transporte de ânion com o aumento da porcentagem de colesterol, indicando, portanto, que a MeBUS[6] era de fato um carreador de ânion, uma vez que o macrociclo precisava se deslocar na membrana para promover o transporte. Além disso, concluiu-se que os cátions não apresentavam nenhum efeito tanto no mecanismo de transporte quanto na seletividade, sugerindo um transporte de ânions através de mecanismos ânion-ânion antiporte ao invés de uma troca simultânea de cátion e ânion. $\mathrm{O}$ trabalho de Reany e colaboradores mostrou que as BU[n] apresentam a característica de um transportador aniônico eficaz, propriedade que deve ser estudada futuramente em sistemas biológicos.

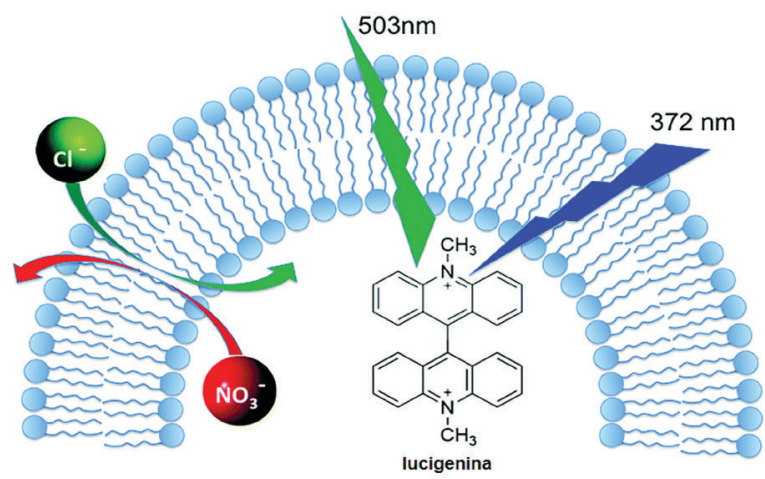

Figura 9. Representação esquemática do influxo de íon cloreto e efluxo de íon nitrato através de vesículas de POPC, monitorada pela fluorescência da lucigenina. Reproduzido parcialmente com permissão da The Royal Society of Chemistry (2017, v. 53, p. 7558)

\section{Sistemas supramoleculares}

Sistemas complexos e funcionais em que os componentes são unidos e organizados por interações não-covalentes constituem a base da química supramolecular. ${ }^{63}$ Sistemas supermoleculares são obtidos a partir de interações eletrostática, hidrofóbica, ligação de hidrogênio ou empilhamento $\pi$ entre moléculas distintas (blocos de construção) e apresentam propriedades diferentes que a mera soma das propriedades individuais de seus componentes. ${ }^{64}$ Uma série de sistemas supramoleculares foram apresentados na literatura, dos quais se destacam os rotaxanos, ${ }^{65}$ as estruturas auto-organizadas ${ }^{66} \mathrm{e}$ os macrociclos supermoleculares.$^{67}$ Esses sistemas supramoleculares encontram uma série de aplicações como sensores químicos ${ }^{68} \mathrm{e}$ biológicos, ${ }^{69}$ catalisadores,${ }^{70}$ carreadores ${ }^{71}$ ou materiais eletrocrômicos e em eletrônica molecular por exemplo. ${ }^{72}$

Na literatura são reportados alguns complexos supramoleculares contendo $\mathbf{B U}[\mathbf{n}]$, para além dos apresentados nesta revisão até este ponto. Sindelar e colaboradores ${ }^{73}$ verificaram a formação de um complexo supramolecular estável de BnBU[6] com íons benzoato e tosilato em clorofórmio contendo resíduo de água. Os dados de difração de raios-X de monocristal indicaram que os ânions em questão não são incluídos na cavidade do macrociclo, uma vez que esses hóspedes são relativamente grandes. Foi verificado, no entanto, que os íons benzoato ou tosilato formam complexos com a BnBU[6] na proporção de 2:1 unidos por ligações de hidrogênio com uma molécula de água no centro da cavidade do macrociclo (Figura 10). Essa função da água como mediador da complexação em solventes apolares úmidos ou no estado sólido já havia sido verificada antes na formação de cápsulas moleculares com outros macrociclos, como os resorcin[n]arenos e pirogalol[n]arenos, ${ }^{74} \mathrm{e}$ indica que as $\mathbf{B U}[\mathbf{n}]$ apresentam potencial para aplicações similares.

Sindelar e colaboradores ${ }^{75}$ tentaram sintetizar caviplexos com BnBU[6] e diversos diaquil fosfatos. Diferentemente do complexo

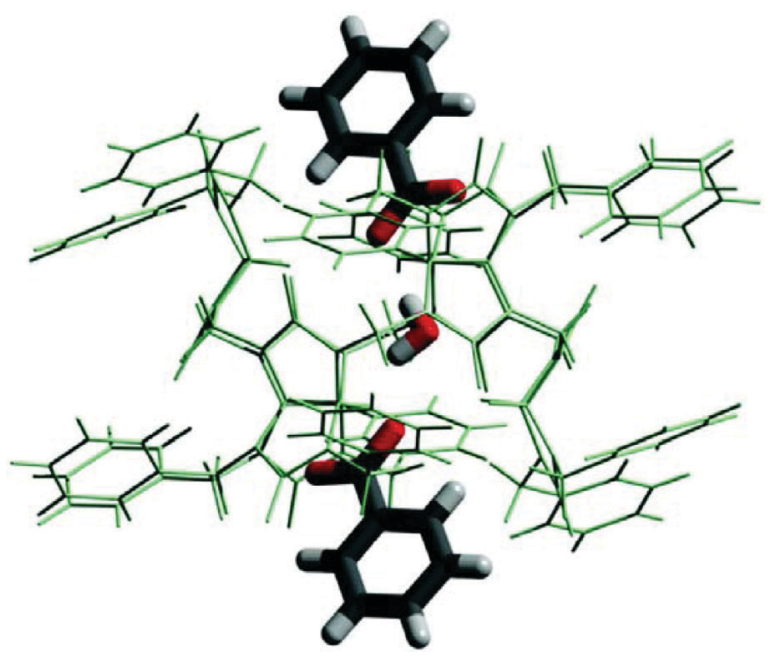

Figura 10. Visão lateral da estrutura cristalina do complexo de íons benzoato com a BnBU[6] na proporção 2:1. Reproduzido com permissão da The Royal Society of Chemistry (2014, v. 50, p. 1372)

anterior, o grupamento fosfato pode ser incluído no interior do macrociclo na proporção 1:1 em duas configurações distintas em solução: internamente, de forma que os substituintes alquila fiquem voltados para fora em sentido oposto, ou externamente, com o grupamento fosfato parcialmente incluído e os substituintes voltados para fora no mesmo sentido. A configuração do tipo externa fez com que os autores estudassem a capacidade das BU[n] de formarem rotaxano, sendo o macrociclo o rotor e os derivados de íons fosfatos os eixos. Algo interessante é que poucos rotaxanos baseados na inclusão de espécies aniônicas em macrociclos foram reportados na literatura até o presente momento, ${ }^{75-78}$ sendo a formação de rotaxanos com BU[n] algo no mínimo incomum.

Baseado nisso, foram realizados experimentos de inclusão de fosfatos aniônicos contendo grupos alcinos terminais como substituintes e posterior reação de condensação com azidas substituídas, na presença de brometo de cobre (I), para a formação dos respectivos triazóis. O objetivo dos autores era verificar se os triazóis e a BnBU[6] formavam rotaxanos e como o tamanho do subtituinte dos íons azida influenciaria na estabilidade desses sistemas supramoleculares (Figura 11). Os resultados de RMN de ${ }^{1} \mathrm{H}$ mostraram que os triazóis
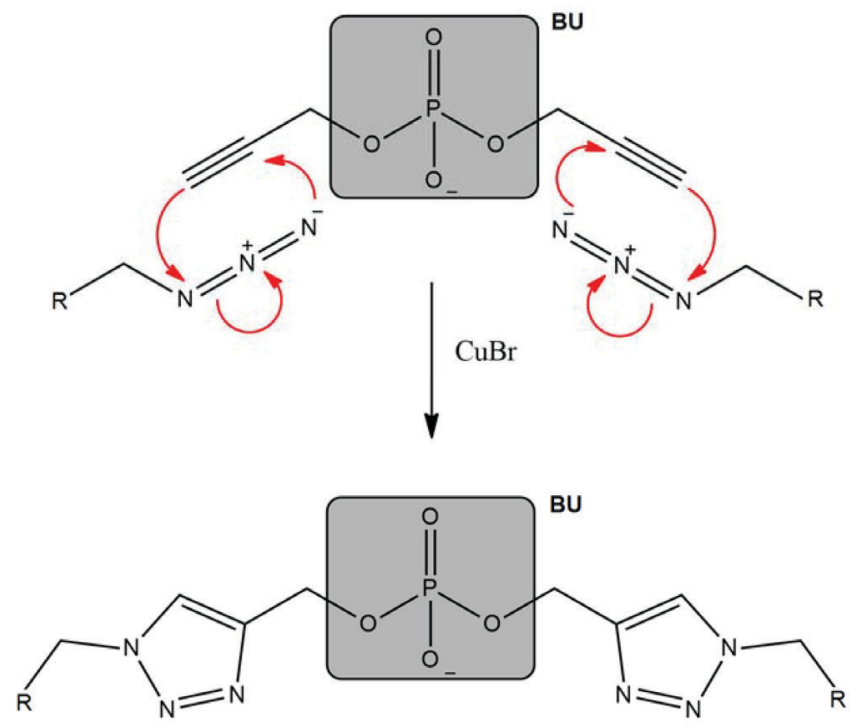

Figura 11. Representação esquemática da formação de um rotaxano de bambusurila (retângulo) e triazol por reação de condensação 
com substituintes pouco volumosos, como etila e propila, podiam se desligar do macrociclo, enquanto os mais volumosos, como trifenilmetila, não permitiam a descomplexação da supermolécula em solução, o que em princípio pareceu promissor.

No entanto, análises por difração de raios-X mostraram que somente a BnBU[6] com moléculas de solvente era obtida no estado sólido, sem nenhum sinal dos derivados de íon fosfato. Experimentos similares realizados com a MeBU[6] indicaram a formação de complexos com estequiometria 2:1 de dialquil-fosfato/ bambusurila ligados pelos grupos fosfato em posição distal a cada opérculo externamente à cavidade. Esses resultados fizeram com que os autores excluíssem a possibilidade de utilizar as BU[n] em estruturas interligadas, mas abrem espaço para outras tentativas com outras moléculas hóspedes.

\section{CONSIDERAÇÕES FINAIS}

Em menos de oito anos, desde que foram sintetizadas pela primeira vez, as bambus[n]urilas já ganharam um lugar de destaque entre os cavitandos, ocupando cada vez mais espaço na literatura especializada em química de inclusão. Isso se explica, em grande parte, pela sua capacidade de complexar ânions de forma seletiva e com elevadas constantes de formação. Não restam dúvidas que esse macrociclo é tão promissor e importante quanto as ciclodextrinas, cucurbiturilas ou pirogalolarenos, e que viabilizará o desenvolvimento de aplicações nos diversos segmentos da química supramolecular. Até este momento, foram publicados cerca de quarenta artigos sobre as bambusurilas, o que mostra que ainda há muito a ser estudado sobre o assunto.

A presente revisão teve como objetivo apresentar a família das bambus[n]urilas, suas propriedades e algumas de suas potenciais aplicações. O escopo da revisão é bastante limitado, mas isso se deve principalmente à escassez de trabalhos na literatura. Ainda existem muitas vacâncias na literatura específica com relação às bambus[n] urilas, como por exemplo, seu uso em catálise, a estabilidade química e física desses macrociclos e a capacidade de estocarem e transportarem fármacos em sistema do tipo "drug delivery". Pouco se sabe ainda sobre a dinâmica de inclusão de moléculas neutras, e acerca da natureza das interações no seio da cavidade. Não se sabe, por exemplo, qual a contribuição de interações dipolares, solvofóbicas, das ligações de hidrogênio e demais forças para a inclusão desses hóspedes. Trabalhos com gases ainda são inexistentes, e não há registro ainda de materiais que a utilizem em aplicações no estado sólido. Uma das limitações a ser superada é a dificuldade de síntese desses compostos, uma vez que são vários os passos e os rendimentos ainda são bem pequenos.

A maioria dos estudos realizados com as bambusurilas são originários da República Tcheca, onde foram reportadas pela primeira vez. Outros estudos têm surgido na China, Israel, Portugal, Índia, Uruguai e França, e aqui no Brasil, nosso grupo tem se dedicado ao estudo das propriedades fundamentais das bambusurilas, suas características eletroquímicas e de coordenação. Acreditamos que uma revisão em língua portuguesa sobre o assunto pode abrir novas perspectivas de pesquisa à nossa comunidade.

\section{AGRADECIMENTOS}

Os autores agradecem à CNPq proc. 309570/2015-8, CNPq proc. 134552/2015-6, FAPESP proc. 2016/12666-1 e FAPESP 2017/195955 pelo financiamento das pesquisas.

\section{REFERÊNCIAS}

1. Jiou, J.; Chiravuri, K.; Gudapati, A.; Gassensmith, J. J.; Curr. Org. Chem. 2014, 18, 2002.
2. Atwood, J. L.; Lehn, J.-M.; Comprehensive Supramolecular Chemistry, Pergamon: Oxford, 1996.

3. Buschmann, H.-J.; Cleve, E.; Jansen, K.; Wego, A.; Schollmeyer, E.; Mater. Sci. Eng., C 2003, 6, 531.

4. Demets, G. J.-F.; Quim. Nova 2007, 30, 1313.

5. Reddy, K. R. K. K.; Cavallini, T. de S.; Demets, G. J.-F.; Silva Jr., L. F.; New J. Chem. 2014, 38, 2262.

6. de Lima, S. M.; Gomez, J. A.; Barros, V. P.; Vertuan, G. de S.; Assis, M. D.; Graeff, C. F. O.; Demets, G. J. F.; Polyhedron 2010, 29, 3008.

7. Chapin, J. C.; Kvasnica, M.; Purse, B. W.; J. Am. Chem. Soc. 2012, 134, 15000 .

8. Bagatin, I. A.; Cruz, A. T.; Toma, H. E.; Politi, M. J.; Demets, G. J.-F.; J. Incl. Phenom. Macrocyc. Chem. 2005, 52, 189.

9. Szejtli, J.; Chem. Rev. 1998, 98, 1743.

10. Holliday, B. J.; Mirkin, C. A.; Angew. Chem., Int. Ed. 2001, 40, 2022.

11. Svec, J.; Necas, M.; Sindelar, V.; Angew. Chem., Int. Ed. 2010, 49, 2378.

12. Svec, J.; Dusek, M.; Fejfarova, K.; Stacko, P.; Klán, P.; Kaifer, A.E.; Li, W.; Hudeckova, E.; Sindelar, V.; Chem. Eur. J. 2011, 17, 5605.

13. Busschaert, N.; Caltagirone, C.; Rossom, W. V.; Gale, P. A.; Chem. Rev. 2015, 115, 8038.

14. Davis, J. T.; Gale, P. A.; Okunola, O. A.; Prados, P.; Sanchez, J. C. I.; Nat. Chem. 2009, 1, 138.

15. Spence, G. T.; Beer, P. D.; Acc. Chem. Res. 2013, 46, 571.

16. Li, Y.; Flood, A. H.; Angew. Chem. 2008, 47, 2649.

17. Correia, H. D.; Cicolani, R. S.; Moral, R. F.; Demets, G. J.-F.; Synthesis 2016, 48, 210.

18. Miyahara, Y.; Goto, K.; Oka, M.; Inazu, T.; Angew. Chem., Int. Ed. 2004, 43, 5019.

19. Buschmann, H.-J.; Jansen, K.; Schollmeyer, E.; Inorg. Chem. Commun. 2003, 6, 531.

20. Cova, T. F. G. G.; Nunes, S. C. C.; Valente, A. J. M.; Melo, T. M. V. D. P.; Pais, A. A. C. C.; J. Mol. Liq. 2017, 242, 640.

21. Révész, A.; Schroder, D.; Svec, J.; Wimmerová, M.; Sindelar, V.; J. Phys. Chem. A 2011, 115, 11378.

22. Cicolani, R. S.; Dissertação de Mestrado, Universidade de São Paulo, Brasil, 2017, 98 p.

23. Gobre, V. V.; Dixit, P. H.; Khedkar, J. K.; Gejji, S. P.; Comput. Theor. Chem. 2011, 976, 76.

24. Toman, P.; Makrlík, E.; Vañura, P.; Acta Chim. Slov. 2011, 58, 846.

25. Toman, P.; Makrlík, E.; Vañura, P.; Chem. Phys. Lett. 2012, 547, 63.

26. Toman, P.; Makrlík, E.; Vañura, P.; Monatsh. Chem. 2012, 143, 985.

27. Toman, P.; Makrlík, E.; Vañura, P.; Acta Chim. Slov. 2013, 60, 174.

28. Makrlík, E.; Toman, P.; Vañura, P.; Monatsh. Chem. 2014, 145, 877.

29. Makrlík, E.; Toman, P.; Vañura, P.; Monatsh. Chem. 2015, 146, 1609.

30. Toman, P.; Makrlík, E.; Vañura, P.; Monatsh. Chem. 2012, 143, 373.

31. Toman, P.; Makrlík, E.; Vañura, P.; Monatsh. Chem. 2012, 143, 1368.

32. Makrlík, E.; Toman, P.; Vañura, P.; Monatsh. Chem. 2014, 145, 721.

33. Cova, T. F. G. G.; Nunes, S. C. C.; Pinho e Melo, T. M. V. D.; Pais, A. A. C. C.; Chem. Phys. Lett. 2017, 672, 89.

34. Havel, V.; Svec, J.; Wimmerova, M.; Dusek, M; Pojarova, M; Sindelar, V.; Org. Lett. 2011, 13, 4000.

35. Havel, V.; Sindelar, V.; ChemPlusChem 2015, 80, 1601.

36. Rivollier, J.; Thuéry, P.; Heck, M-P.; Org. Lett. 2013, 15, 480.

37. Yawer, M. A.; Havel, V.; Sindelar, V.; Angew. Chem., Int. Ed. 2015, 54, 276.

38. Havel, V.; Babiak, M.; Sindelar, V. Chem. Eur. J. 2017, 23, 8963.

39. Singh, M.; Solel, E.; Keinan, E.; Reany, O.; Chem. Eur. J. 2015, 21, 536.

40. Singh, M.; Solel, E.; Keinan, E.; Reany, O.; Chem. Eur. J. 2016, 22 , 8848 .

41. Solel, E.; Singh, M.; Reany, O.; Keinan, E.; Phys. Chem. Chem. Phys. 2016, $18,13180$.

42. Gerasko, O. A.; Fedin, V. P.; Russ. J. Inorg. Chem. 2011, 56, 2025.

43. Choi, K. H.; Hamilton, A. D.; Coord. Chem. Rev. 2003, 240, 101 
44. Gonzalez, A. D.; Hoepfl, H.; Medrano, F.; Yatsimirsky, A. K.; J. Org. Chem. 2010, 75, 2259.

45. Chang, S. G.; Littlejohn, D.; Hu, K. Y.; Science 1987, 237, 756.

46. Evans, N. H.; Beer, P. D.; Angew. Chem., Int. Ed. 2014, 53, 11716.

47. Langton, M. J.; Robinson, S. W.; Marques, I.; Félix, V.; Beer, P. D. ; Nat Chem. 2014, 6, 1039.

48. Havel, V.; Yawer, M. A.; Sindelar, V.; Chem. Commun. 2015, 51, 4666.

49. Silab, S. D.; Doran, S.; Yagci, Y.; Chem. Rev. 2016, 116, 10212.

50. Yoon, K. B.; Kochi, J. K.; J. Am. Chem. Soc. 1988, 110, 6586.

51. Alvaro, M.; Bizzoca, G.; Ferrer, B.; Garcia, H.; de Miguel, M.; Teruel, L.; ChemPhysChem 2010, 11, 3456.

52. Alvaro, M.; Carbonell, E.; Ferrer, B.; Llabrés, X. F. X.; Garcia, H. Chemistry 2017, 13, 5106.

53. Fiala, T.; Ludvikova, L.; Heger, D.; Svec, J.; Slanina, T.; Vetrakova L.; Babiak, M.; Necas, M.; Kulhanek, P.; Klan, P.; Sindelar, V.; J. Am. Chem. Soc. 2017, 139, 2597.

54. Wright, S. H.; Dantzler, W. H.; Physiol. Rev. 2004, 84, 987.

55. Cametti, M.; Rissanen, K.; Chem. Commun. 2009, 20, 2809.

56. Jentsch, T. J.; Maritzen, T.; Zdebik, A. A.; J. Clin. Invest. 2005, 115, 2039.

57. Delange, F.; Thyroid 1994, 4, 107.

58. Evans, N. H.; Beer, P. D.; Angew. Chem., Int. Ed. 2014, 53, 11716.

59. Patel, N.; Ramachandran, S.; Azimov, R.; Kagan, B. L.; Lal, R.; Biochemistry 2015, 54, 7320.

60. Gale, P. A.; -Tomás, R. P.; Quesada, R.; Acc. Chem. Res. 2013, 46, 2801.

61. Gale, P. A.; Acc. Chem. Res. 2011, 44, 216.

62. Lang, C.; Mohite, A.; Deng, X.; Yang, F.; Dong, Z.; Xu, J.; Liu, J.; Keinan, E.; Reany, O. Chem. Commun. 2017, 53, 7557.
63. Lehn, J. -M.; Chem. Soc. Rev. 2007, 36, 151

64. Bohne, C.; Chem. Soc. Rev. 2014, 43, 4037.

65. Xue, M.; Yang, Y.; Chi, X.; Yan, X.; Huang, F.; Chem. Rev. 2015, 115, 7398.

66. Lehn, J.-M.; Angew. Chem., Int. Ed. Engl. 1990, 29, 1304.

67. Lindoy, L. F.; Park, K. M.; Lee, S. S.; Chem. Soc. Rev. 2013, 42, 1713.

68. Oshovsky, G. V.; Reinhoudt, D. N.; Verboom, W.; Angew. Chem., Int. Ed. 2007, 46, 2366

69. Daze, K. D.; Pinter, T.; Beshara, C. S.; Ibraheem, A.; Minaker, S. A.; Ma, M. C. F.; Courtemanche, R. J. M.; Campbell, R. E.; Hof, F.; Chem. Sci. 2012, 3, 2695.

70. Hirst, A. R.; Roy, S.; Arora, M.; Das, A. K.; Hodson, N.; Murray, P.; Marshall, S.; Javid, N.; Sefcik, J.; Boekhoven, J.; Esch, J. H. van.; Santabarbara, S.; Hunt, N. T.; Ulijin, R. V.; Nat. Chem. 2010, 2, 1089.

71. Lehn, J.-M.; Science. 1985, 227, 849.

72. Schott, M.; Lorrmann, H.; Szczerba, W.; Beck, M.; Kurth, D. G.; Sol. Energy Mater. Sol. Cells 2014, 126, 68.

73. Havel, V.; Sindelar, V.; Necas, M.; Kaifer, A. E.; Chem. Commun. 2014, 50, 1372.

74. Avram, L.; Cohen, Y.; Rebek, J. Jr.; Chem. Commun. 2011, 47, 5368.

75. Fiala, T.; Sindelar, V.; Supramol. Chem. 2016, 28, 810.

76. Ghosh, P.; Mermagen, O.; Schalley, C. A.; Chem. Commun. 2002, 22 , 2628.

77. Li, Y.; Flood, A. H.; Angew. Chem., Int. Ed. 2008, 47, 2649.

78. Lee, S.; Chen, C. -H.; Flood, A. H.; Nat. Chem. 2013, 5, 704. 